\title{
Epidemiologia de micoses, pitiose e micotoxicoses em equinos no sudeste do Rio Grande do Sul ${ }^{1}$
}

\author{
Angelita Reis-Gomes ${ }^{2 *}$, Clairton Marcolongo-Pereira ${ }^{3}$, Eliza Simone V. Sallis ${ }^{4}$, \\ Fábio R.P. Bruhn ${ }^{5}$, Renata O. Faria ${ }^{5}$, Ana Lucia Schild ${ }^{6}$ e Mario C.A. Meireles ${ }^{5}$
}

\begin{abstract}
Reis-Gomes A., Marcolongo-Pereira C., Sallis E.S.V., Bruhn F.R.P., Faria R.O., Schild A.L. \& Meireles M.C.A. 2018. [Epidemiology of mycoses, pitiosis and micotoxicosis in horses in southeastern Rio Grande do Sul, Brazil.] Epidemiologia das micoses, pitiose e micotoxicoses em equinos no sudeste do Rio Grande do Sul. Pesquisa Veterinária Brasileira 38(6):1110-1116. Laboratório Regional de Diagnóstico, Faculdade de Veterinária, Universidade Federal de Pelotas, Campus Universitário, Capão do Leão, RS 96010-900, Brazil. E-mail: angelitagomes@gmail.com

The epidemiological aspects of diseases caused by fungi and oomycetes in horses in southeastern Rio Grande do Sul, Brazil, is described. The epidemiological rates, their causes, and health importance in the region were established. A descriptive epidemiology study was carried out in relation of potential risk factors. The impact on these diseases in the region was measured. From 1978 to 2014, pythiosis had a prevalence of 49.71\% (86/173), and mycotoxicoses of $30.05 \%$ (52/173), with 45 cases of leukoencephalomalacia and 7 of ergotism. The prevalence of fungal infections was $19.65 \%$ (34/173) of cases. Dermatophytosis was the most prevalent fungal infection with 58.82\% (20/34) of cases. The most isolated dermatophyte species were Trichophyton mentagrophytes $60 \%(12 / 20)$, Trichophyton equinum $25 \%$ (5/20) and Microsporum gypseum, Microsporum canis, and Trichophyton verrucosum, both responsible for 5\% (1/20) of infections. Rhinosporidose was diagnosed in $35.29 \%$ $(12 / 34)$ of cases. Uterine mycosis caused by Candida albicans and Cryptococcus laurentii was observed at 5.88\% (2/34) of cases. Cladosporium sp. allergy was noted in one record. According to the incidence from 1990 to 2014, pythiosis had median incidence (MI) of 2.98 and interquartile range (DI) of 3.82. Mares were 4.18 times likely to develop the disease then males. The disease occurs in the region in every season. Leukoencephalomalacia had MI of 0.0; DI 1.00 and male horses were 3.4 times more likely than mares to develop the disease. Leukoencephalomalacia was 6 times more likely to occur during winter. Ergotism had MI of 0.00 ; DI of 0.000 , rhinosporidiosis MI of 0.00 , DI of 0.088 and ringworm MI of 0.00 , and DI of 0.935 . In the study pythiosis had the highest prevalence among the diseases observed, and may be considered endemic in the region. The magnitude of the diseases observed may be even greater within the equine herd, since these diseases are not of obligatory notification and some are well known by veterinarians and owners, who often do not obtain a laboratory confirmation of the diagnosis.
\end{abstract}

INDEX TERMS: Epidemiology, mycoses, pitiosis, micotoxicosis, horses, Rio Grande do Sul.

\footnotetext{
${ }^{1}$ Recebido em 17 de maio de 2017.

Aceito para publicação em 31 de maio de 2017.

${ }^{2}$ Programa de Pós-Graduação em Veterinária, Faculdade de Veterinária (FV), Universidade Federal de Pelotas (UFPel), Campus Universitário, Pelotas, RS 96010-900, Brasil.

${ }^{3}$ Faculdade de Medicina Veterinária, Centro Universitário Ritter dos Reis (UniRitter), Porto Alegre, RS 90840-440, Brasil.
}

\footnotetext{
${ }^{4}$ Docente do Departamento de Patologia Animal, Faculdade de Veterinária (FV), Universidade Federal de Pelotas (UFPel), Pelotas, RS, Brasil 96010-900.

${ }^{5}$ Docente do Departamento de Veterinária Preventiva, Faculdade de Veterinária (FV), Universidade Federal de Pelotas (UFPel), Pelotas, Brasil, RS 96010-900.

${ }^{6}$ Laboratório Regional de Diagnóstico, Faculdade de Veterinária(FV), Universidade Federal de Pelotas (UFPel), Campus Universitário Capão do Leão, Pelotas, RS 96010-900, Brasil. *Autor para correspondência: angelitagomes@gmail.com
} 
RESUMO.- Descrevem-se os aspectos epidemiológicos das doenças causadas por fungos e oomicetos na população de equinos na região sudeste do Rio Grande do Sul, estabelecendo as taxas epidemiológicas, suas causas e sua importância sanitária na região. Foi realizada a epidemiologia descritiva por meio do cálculo da incidência das doenças encontradas ao longo dos anos e verificada a existência de associação entre a ocorrência dessas enfermidades e o sexo, a raça e a estação do ano. Entre os anos de 1978 e 2014 a pitiose teve prevalência de 49,71\% (86/173), as micotoxicoses 30,05\% (52/173), sendo 45 casos de leucoencefalomalácia e sete de ergotismo. As micoses tiveram prevalência de 19,65\% (34/173), sendo as dermatofitoses as mais prevalentes com 58,82\% (20/34) dos casos. As espécies de dermatófitos mais frequentemente isoladas foram Trichophyton mentagrophytes $60 \%(12 / 20)$, Trichophyton equinum $25 \%$ (5/20) e Microsporum gypseum, Microsporum canis e Trichophyton verrucosum ambos responsáveis por 5\% (1/20) das infecções. Rinosporidiose foi diagnosticada em 35,29\% (12/34) dos casos. Micoses uterinas causadas por Candida albicans e Cryptococcus laurentii foram observadas em 5,88\% (2/34) dos casos. Alergia por Cladosporium sp. teve um registro. De acordo com as incidências calculadas entre 1990 e 2014 a pitiose teve incidência mediana (IM) 2,98 e distância interquartil (DI) $=3,82$, as fêmeas tiveram chance 4,18 vezes maiores de desenvolver a doença, a enfermidade ocorre independente das estações climáticas. A leucoencefalomalácia teve IM=0,0; DI 1,00 e equinos machos tiveram 3,4 vezes mais chance de desenvolver a doença que fêmeas, no inverno a possibilidade de ocorrência dessa enfermidade foi seis vezes maior. 0 ergotismo teve $\mathrm{IM}=0,00 ; \mathrm{DI}=0,000$, rinosporidiose $\mathrm{IM}=0,00 ; \mathrm{DI}=0,088$ e dermatofitose $\mathrm{IM}=0,00 ; \mathrm{DI}=0,935$. A pitiose foi mais prevalente entre as doenças encontradas, podendo ser considerada endêmica na região. Considera-se que a magnitude das doenças possa ser ainda maior dentro do rebanho equino, uma vez que as doenças descritas não são de notificação obrigatória e algumas são bem conhecidas por veterinários e proprietários, que muitas vezes não fazem a confirmação laboratorial do diagnóstico.

TERMOS DE INDEXAÇÃO: Epidemiologia, doenças fúngicas, pitiose, micotoxicoses, micoses, equinos, Rio Grande do Sul.

\section{INTRODUÇÃO}

Doenças infecciosas causadas por fungos e oomicetos são consideradas ameaças à diferentes espécies. A ação de fungos em plantas, animais e humanos representa perdas de produção e sérios problemas de saúde, principalmente em humanos e animais imunossuprimidos (CAST 2003).

Nas últimas décadas o número de doenças em animais causadas por fungos tem aumentado, novas espécies patogênicas têm sido identificados, e sua dispersão tem sido facilitada pela modificação do meio ambiente (Fisher et al. 2012). Contudo, em medicina veterinária o progresso para compreender e desenvolver os meios para prevenção e controle das doenças fúngicas tem sido mais lento que na medicina humana. Muitas doenças fúngicas não são diagnosticadas até o exame post mortem (Blanco \& Garcia 2008).

Estudos epidemiológicos sobre doenças fúngicas são escassos, não havendo informações precisas sobre a prevalência e incidência dessas enfermidades ou sobre variações dos fatores de risco envolvidos. Por não serem de notificação obrigatória, há pouca informação disponível sobre essas enfermidades, o que aumenta o desafio diagnóstico, dificulta a identificação e caracterização dos agentes etiológicos e as características clínico-patológicas dessas doenças. Ainda, é necessário considerar o impacto em saúde pública dessas enfermidades, uma vez que algumas dessas são zoonoses.

Em equinos, doenças fúngica são em sua maioria estudadas através de relatos de casos isolados, não havendo muitos estudos epidemiológicos em populações (Scott \& Miller 2011, Cafarchia et al. 2013). Os objetivos deste estudo foram estabelecer a epidemiologia das principais doenças fúngicas ocorridas em equinos na região sudeste do Rio Grande do Sul, entre os anos de 1978 e 2014, assim como determinar a incidência e os fatores de risco associados, estabelecendo um panorama epidemiológico dessas enfermidades na região.

\section{MATERIAL E MÉTODOS}

Foi realizado um estudo retrospectivo das doenças causadas por fungos e oomicetos em equinos diagnosticadas pelo Laboratório Regional de Diagnóstico (LRD) da Faculdade de Veterinária, Universidade Federal de Pelotas (UFPel), entre 1978 e 2014.0 estudo foi baseado em consulta e avaliação dos protocolos de necropsia e laudos micológicos arquivados pelo LRD/UFPel. Para o estudo epidemiológico descritivo foram coletadas informações relativas a idade, a raça, ao sexo, às estações do ano e à origem dos casos. A normalidade na distribuição dos casos registrados foi testada por meio de testes de Shapiro-Wilk. Considerando a ausência de normalidade na distribuição de incidências ao longo dos anos optou-se pelo uso da mediana e a sua distância interquartílica para descrição das incidências das diferentes doenças. 0 cálculo da taxa de incidência anual das enfermidades foi realizado de forma estratificada, representativo da área de abrangência do LRD/UFPel. Para o cálculo das taxas de incidência, foram utilizados dados relativos ao censo anual da população de equinos na mesorregião sudeste do Rio Grande do Sul, fornecidos pelo Instituto Brasileiro de Geografia e Estatística (IBGE), que dispunha das informações censitárias de equinos a partir do ano de 1990 até 2014. Para avaliar a associação entre idade, raça, sexo e estação do ano e a ocorrência das diferentes enfermidades diagnosticadas em equinos, foram aplicados testes de qui-quadrado ou exato de Fisher (esse quando ocorreram menos que cinco observações em pelo menos uma casela na tabela de contingência $2 \times 2$ ). 0 risco nessas associações foi estimado por meio da Odds Ratio e seu intervalo de confiança a 95\% (IC. 95\%), para as variáveis que apresentaram associação significativa $(\mathrm{p}<0,05)$ nos testes de Qui-quadrado. Considerou-se um valor mínimo de confiança de 95\% em todas as análises estatísticas, as quais foram realizadas por meio dos softwares SPSS20.0 e Epilnfo 7.1.5.2.

\section{RESULTADOS}

No período do estudo foram recebidos para análise no LRD/UFPel 2210 cadáveres e\ou materiais biológicos de equinos, dos quais 173 resultaram em diagnósticos positivos para doenças fúngicas. Destes, a maior parte dos animais era criada no município de Pelotas $(60,7 \%)$, seguida por Bagé (5,8\%), Jaguarão, Rio Grande, Santa Vitória do Palmar (4,6\% em cada), São Lourenço do Sul (4\%) e Capão do Leão (2,3\%). Os municípios de São José do Norte, Piratini, Arroio Grande, Pedro Osório, Pinheiro Machado, Canguçu, Morro Redondo, Cristal, Aceguá, Camaquã, Candiota, Tapes e Santana 
da Boa Vista apresentaram, em conjunto, 13,3\% dos casos diagnosticados.

Dentre os 173 equinos, 50,87\% eram fêmeas, 32,37\% machos e em $23,70 \%$ dos protocolos não havia essa informação. A maioria dos animais tinha idade acima de 5 anos $(45,09 \%)$, $31,21 \%$ eram menores de 5 anos, em $23,70 \%$ dos protocolos não havia informação sobre a idade. A raça crioula foi a mais afetada com $51,45 \%$ dos casos, $34,10 \%$ eram animais sem raça definida e 6,94\% Puro Sangue Inglês. As demais raças ficaram abaixo de $3 \%$ da casuística.

A prevalência das enfermidades, entre os anos de 1978 e 2014, conforme as variáveis idade, sexo, estações do ano e raça podem ser observadas no Quadro 1.

No Quadro 2 encontram-se as incidências anuais (por 100.000 equinos) dos grupos de doenças causadas por fungos, oomicetos e micotoxicoses diagnosticadas na região sudeste do Rio Grande do Sul entre os anos de 1990 e 2014. Dentre as enfermidades analisadas, a pitiose foi a doença mais prevalente em equinos na região, representando $49,71 \%$ (86/173) dos casos, com distribuição equilibrada ao longo dos anos, e incidências variando de 0 a 8,15 (Quadro 2), apresentando incidência mediana (IM) de 2,98 e distância interquartil (DI) de 3,82. Analisando equinos com enfermidades fúngicas, sexo e raça demonstraram ser fatores associados ao desenvolvimento de pitiose (Quadro 3), sendo que fêmeas ( $p=0,000 ; 0 R=4,18 ; I C, 95 \%=1,919$ - 9,158), e animais com raça definida tiveram maior chance de apresentar a enfermidade ( $p=0,000 ; 0 R=4,52 ; \mathrm{IC}, 95 \%=2,264-9,035)$.

As micotoxicoses tiveram prevalência de 30,05\% (52/173). Dessas, a leucoencefalomalacia equina (LEME) (45/52) foi a mais frequente, tendo entre os anos de 1990 e 2014, IM=0,0 (DI= 1,00 ) (Quadro2). Machos tiveram maior chance de apresentar a doença que as fêmeas $(p=0,004)$, assim como a ocorrência nos meses mais frios em relação aos mais quentes $(p=0,000)$. Animais sem raça definida também tiveram maior chance de desenvolver LEME ( $p=0,000)$ (Quadro 4). Dos sete casos de ergotismo, cinco ocorreram no inverno de 1986 e dois na primavera de 2008 (Quadro 1), sem associação significativa entre os casos e o sexo $(\mathrm{p}=0,830)$, idade $(\mathrm{p}=0,364)$, estação climática $(\mathrm{p}=0,884)$ e raça $(\mathrm{p}=0,242)$.

As micoses tiveram prevalência de 19,65\% (34/173). A dermatofitose foi a micose mais frequente com $58,82 \%(20 / 34)$

Quadro 1. Frequência das doenças causadas por fungos, oomicetos e micotoxinas diagnosticadas em equinos na região sudeste do Rio Grande do Sul, 1978-2014

\begin{tabular}{|c|c|c|c|c|c|c|c|c|c|c|c|c|}
\hline \multirow{3}{*}{ Fatores de risco } & \multicolumn{12}{|c|}{ Enfermidade } \\
\hline & \multicolumn{2}{|c|}{ LEME } & \multicolumn{2}{|c|}{ Ergotismo } & \multicolumn{2}{|c|}{ Dermatofitose } & \multicolumn{2}{|c|}{ Rinosporidiose } & \multicolumn{2}{|c|}{ Pitiose } & \multicolumn{2}{|c|}{ TOTAL } \\
\hline & $\mathrm{N}$ & $\%$ & $\mathrm{~N}$ & $\%$ & $\mathrm{~N}$ & $\%$ & $\mathrm{~N}$ & $\%$ & $\mathrm{~N}$ & $\%$ & $\mathrm{~N}$ & $\%$ \\
\hline \multicolumn{13}{|l|}{ Idade em anos } \\
\hline $0-5$ & 12 & 26,7 & 4 & 57,1 & 7 & 35,0 & 2 & 16,7 & 28 & 32,6 & 54 & 31,21 \\
\hline$>5$ & 16 & 35,6 & 3 & 42,9 & 8 & 40,0 & 4 & 33,3 & 46 & 53,5 & 78 & 45,09 \\
\hline NI & 17 & 37,8 & 0 & 0,0 & 5 & 25,0 & 6 & 50,0 & 12 & 14,0 & 41 & 23,70 \\
\hline Total & 45 & 100,0 & 7 & 100,0 & 20 & 100,0 & 12 & 100,0 & 86 & 100,0 & 173 & 100,00 \\
\hline \multicolumn{13}{|l|}{ Sexo } \\
\hline M & 17 & 37,8 & 3 & 42,9 & 6 & 30,0 & 8 & 66,7 & 21 & 24,4 & 56 & 32,37 \\
\hline $\mathrm{F}$ & 10 & 22,2 & 4 & 57,1 & 8 & 40,0 & 2 & 16,7 & 62 & 72,1 & 88 & 50,87 \\
\hline NI & 18 & 40,0 & 0 & 0,0 & 6 & 30,0 & 2 & 16,7 & 3 & 3,5 & 29 & 16,76 \\
\hline Total & 45 & 100,0 & 7 & 100,0 & 20 & 100,0 & 12 & 100,0 & 86 & 100,0 & 173 & 100,00 \\
\hline \multicolumn{13}{|l|}{ Estação } \\
\hline Primavera & 1 & 2,2 & 5 & 71,4 & 4 & 20,0 & 4 & 33,3 & 3 & 3,5 & 19 & 10,98 \\
\hline Verão & 3 & 6,7 & 0 & 0,0 & 3 & 15,0 & 3 & 25,0 & 15 & 17,4 & 24 & 13,87 \\
\hline Outono & 6 & 13,3 & 0 & 0,0 & 6 & 30,0 & 5 & 41,7 & 39 & 45,3 & 57 & 32,95 \\
\hline Inverno & 35 & 77,8 & 2 & 28,6 & 3 & 15,0 & 0 & 0,0 & 8 & 9,3 & 48 & 27,75 \\
\hline NI & 0 & 0,0 & 0 & 0,0 & 4 & 20,0 & 0 & 0,0 & 21 & 24,4 & 25 & 14,45 \\
\hline Total & 45 & 100,0 & 7 & 100,0 & 20 & 100,0 & 12 & 100,0 & 86 & 100,0 & 173 & 100,00 \\
\hline \multicolumn{13}{|l|}{ Raça } \\
\hline Crioula & 13 & 28,9 & 0 & 0,0 & 11 & 55,0 & 4 & 33,3 & 60 & 69,8 & 89 & 51,45 \\
\hline Cruza & 30 & 66,7 & 1 & 14,3 & 6 & 30,0 & 6 & 50,0 & 16 & 18,6 & 59 & 34,10 \\
\hline Mangalarga & 0 & 0,0 & 2 & 28,6 & 0 & 0,0 & 0 & 0,0 & 0 & 0,0 & 2 & 1,16 \\
\hline Puro Sangue Inglês & 2 & 4,4 & 4 & 57,1 & 1 & 5,0 & 1 & 8,3 & 2 & 2,3 & 12 & 6,94 \\
\hline Percheron & 0 & 0,0 & 0 & 0,0 & 0 & 0,0 & 0 & 0,0 & 4 & 4,7 & 4 & 2,31 \\
\hline Pônei & 0 & 0,0 & 0 & 0,0 & 0 & 0,0 & 1 & 8,3 & 1 & 1,2 & 2 & 1,16 \\
\hline Quarto de milha & 0 & 0,0 & 0 & 0,0 & 1 & 5,0 & 0 & 0,0 & 0 & 0,0 & 1 & 0,58 \\
\hline Árabe & 0 & 0,0 & 0 & 0,0 & 0 & 0,0 & 0 & 0,0 & 2 & 2,3 & 2 & 1,16 \\
\hline NI & 0 & 0,0 & 0 & 0,0 & 1 & 5,0 & 0 & 0,0 & 1 & 1,2 & 2 & 1,16 \\
\hline Total & 45 & 100,0 & 7 & 100,0 & 20 & 100,0 & 12 & 100,0 & 86 & 100,0 & 173 & 100,00 \\
\hline
\end{tabular}

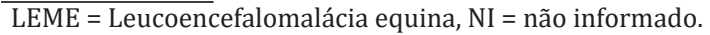


Quadro 2. Incidência anual (por 100.000 equinos) dos grupos de doenças causadas por fungos, oomicetos e micotoxinas diagnosticadas na região sudeste do Rio Grande do Sul, 1990-2014

\begin{tabular}{|c|c|c|c|c|c|c|}
\hline \multirow{2}{*}{ Ano } & \multirow{2}{*}{ População } & \multicolumn{5}{|c|}{ Incidência (por 100.000 equinos) } \\
\hline & & Ergotismo & LEME & Dermatofitose & Rinosporidiose & Pitiose \\
\hline 1990 & 138869 & 0,00 & 4,32 & 2,88 & 0,72 & 0,72 \\
\hline 1992 & 132016 & 0,00 & 0,76 & 1,51 & 0,00 & 0,00 \\
\hline 1993 & 126991 & 0,00 & 0,00 & 0,00 & 0,00 & 0,00 \\
\hline 1994 & 129106 & 0,00 & 0,77 & 0,77 & 0,00 & 0,00 \\
\hline 1996 & 99565 & 0,00 & 3,01 & 0,00 & 0,00 & 4,02 \\
\hline 1997 & 104197 & 0,00 & 0,96 & 0,00 & 0,00 & 3,84 \\
\hline 1998 & 106316 & 0,00 & 0,00 & 1,88 & 0,00 & 3,76 \\
\hline 1999 & 107039 & 0,00 & 0,93 & 0,00 & 0,00 & 5,61 \\
\hline 2003 & 107181 & 0,00 & 0,00 & 0,00 & 0,00 & 1,87 \\
\hline 2004 & 98170 & 0,00 & 0,00 & 0,00 & 0,00 & 8,15 \\
\hline 2005 & 100615 & 0,00 & 0,00 & 0,00 & 0,00 & 2,98 \\
\hline 2006 & 101444 & 0,00 & 0,00 & 1,97 & 0,00 & 2,96 \\
\hline 2007 & 96307 & 0,00 & 1,04 & 0,00 & 1,04 & 2,08 \\
\hline 2008 & 95805 & 1,04 & 1,04 & 1,04 & 1,04 & 5,22 \\
\hline 2009 & 97132 & 0,00 & 0,00 & 1,03 & 0,00 & 7,21 \\
\hline 2010 & 100408 & 0,00 & 0,00 & 0,00 & 1,99 & 4,98 \\
\hline 2011 & 92625 & 0,00 & 0,00 & 0,00 & 1,08 & 4,32 \\
\hline 2012 & 92435 & 0,00 & 0,00 & 0,00 & 1,08 & 3,25 \\
\hline
\end{tabular}

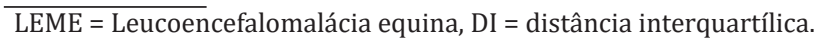

Quadro 3. Associação pelo teste de qui-quadrado entre sexo, idade, estação do ano e raça de equinos com pitiose em relação às outras doenças fungicas diagnosticadas na região sudeste do Rio Grande do Sul, 1978-2014

\begin{tabular}{|c|c|c|c|c|c|}
\hline \multirow{2}{*}{ Fatores } & \multicolumn{2}{|c|}{ Pitiose } & \multirow{2}{*}{ Valor de $\mathrm{p}$} & \multirow{2}{*}{ Odds ratio } & \multirow{2}{*}{ IC (95\%) } \\
\hline & Sim & Não & & & \\
\hline \multicolumn{6}{|l|}{ Sexo } \\
\hline Macho & 21 & 34 & \multirow{2}{*}{0,000} & 1 & \multirow{2}{*}{$1,919-9,158$} \\
\hline Fêmea & 62 & 24 & & 4,182 & \\
\hline \multicolumn{6}{|l|}{ Idade (anos) } \\
\hline $0-5$ & 28 & 25 & \multirow{2}{*}{0,434} & & \\
\hline$>5$ & 46 & 31 & & & \\
\hline \multicolumn{6}{|l|}{ Estação } \\
\hline Outono/inverno & 47 & 57 & \multirow{2}{*}{0,888} & & \\
\hline Primavera/verão & 18 & 23 & & & \\
\hline \multicolumn{6}{|l|}{ Raça } \\
\hline Sem raça definida & 16 & 43 & \multirow{2}{*}{0,000} & 1 & \multirow{2}{*}{$2,264-9,035$} \\
\hline Raça & 69 & 41 & & 4,522 & \\
\hline
\end{tabular}


Quadro 4. Associação pelo teste de qui-quadrado entre sexo, idade, estação do ano e raça de equinos com leucoencefalomalácia em relação às outras doenças fungicas diagnosticadas na região sudeste do Rio Grande do Sul, 1978-2014

\begin{tabular}{|c|c|c|c|c|c|}
\hline \multirow{2}{*}{12} & \multicolumn{2}{|c|}{ Leucoencefalomalácia } & \multirow{2}{*}{ Valor de $\mathrm{p}$} & \multirow{2}{*}{ Odds ratio } & \multirow{2}{*}{ IC (95\%) } \\
\hline & Sim & Não & & & \\
\hline \multicolumn{6}{|l|}{ Sexo } \\
\hline Fêmea & 10 & 76 & \multirow{2}{*}{0,004} & 1 & \multirow{2}{*}{$1,420-8,138$} \\
\hline Macho & 17 & 38 & & 3,4 & \\
\hline \multicolumn{6}{|l|}{ Idade (anos) } \\
\hline $0-5$ & 12 & 41 & 0,799 & & \\
\hline Primavera/verão & 4 & 37 & \multirow{2}{*}{0} & 1 & \multirow{2}{*}{$1,928-24,730$} \\
\hline Outono/inverno & 41 & 63 & & 6,019 & \\
\hline \multicolumn{6}{|l|}{ Raça } \\
\hline Raça definida & 15 & 95 & \multirow{2}{*}{0,000} & 1 & \multirow{2}{*}{$2,922-14,877$} \\
\hline Sem raça definida & 30 & 29 & & 6,55 & \\
\hline
\end{tabular}

Quadro 5. Associação pelo teste de qui-quadrado entre sexo, idade, estação do ano e raça de equinos com rinosporidiose em relação às outras doenças fúngicas diagnosticadas na região sudeste do Rio Grande do Sul, 1978-2014

\begin{tabular}{|c|c|c|c|c|c|}
\hline \multirow{2}{*}{ Fatores } & \multicolumn{2}{|c|}{ Rinosporidiose } & \multirow{2}{*}{ Valor de p } & \multirow{2}{*}{ Odds ratio } & \multirow{2}{*}{ IC (95\%) } \\
\hline & Sim & Não & & & \\
\hline \multicolumn{6}{|l|}{ Sexo* } \\
\hline Fêmea & 2 & 84 & \multirow{2}{*}{0,005} & 1 & \multirow{2}{*}{$1,332-70,833$} \\
\hline Macho & 8 & 47 & & 7,149 & \\
\hline \multicolumn{6}{|l|}{ Idade (anos)* } \\
\hline $0-5$ & 2 & 51 & \multirow{2}{*}{0,704} & & \\
\hline$>5$ & 4 & 73 & & & \\
\hline \multicolumn{6}{|l|}{ Estação } \\
\hline Outono/inverno & 5 & 99 & \multirow{2}{*}{0,015} & 1 & \multirow{2}{*}{$1,024-17,240$} \\
\hline Primavera/verão & 7 & 34 & & 4,076 & \\
\hline \multicolumn{6}{|l|}{ Raça } \\
\hline Raça & 6 & 104 & \multirow{2}{*}{0,255} & & \\
\hline Sem raça definida & 6 & 53 & & & \\
\hline
\end{tabular}

dos casos (Quadro 1), as espécies de dermatófitos isolados foram Trichophyton mentagrophytes 60\% (12/20), Trichophyton equinum 25\% (5/20) e Microsporum gypseum, Microsporum canis e Trichophyton verrucosum ambos responsáveis por $5 \%(1 / 20)$. A dermatofitose teve $\mathrm{IM}=0,00$ (DI=0,935), e não houve associação significativa para os fatores sexo $(p=0,755)$, idade $(\mathrm{p}=0,621)$, estação climática $(\mathrm{p}=0,145)$ e raça $(\mathrm{p}=0,623)$.

A rinosporidiose ocorreu em 35,29\% (12/34) dos casos de micose (Quadro 1), sendo 83,33\% (10/12) após o ano de 2007 , tendo $\mathrm{IM}=0,00$ (DI=0,088). Em relação às outras doenças, machos tiveram mais chance de apresentar rinosporidiose $(\mathrm{p}=0,005)$, e uma maior frequência nos meses da primavera e verão ( $\mathrm{p}=0,015)$ (Quadro 5).

As micoses uterinas corresponderam a 5,88\% (2/34) dos casos, sendo uma causada por Candida albicans e uma por Cryptococcus laurentii. Rinite por Cladosporium sp. teve um registro.

\section{DISCUSSÃO}

Embora não existam informações acerca dos indicadores epidemiológicos da pitiose equina no Brasil, por meio dos dados encontrados confirma-se que a região sudeste do Rio Grande do Sul é uma das regiões do país com maior prevalência, zona endêmica. Conforme Marcolongo-Pereira et al. (2014), a pitiose é uma das principais causas de lesões cutâneas em equinos na região, o que corrobora a IM de 2,8 encontrada no presente estudo, que indica uma característica de endemicidade da doença e justifica a afirmação de Pierezan et al. (2009) que a pitiose representa 3,58\% de causas de mortes equinas na região sul do país.

Considerando que o acúmulo de água em banhados e lagoas influencia a ocorrência da pitiose (Miller \& Campbell 1982), um dos prováveis fatores responsáveis pela maior casuística da região é a localização geográfica na planície costeira, 
constituída em parte pela Bacia de Pelotas, que apresenta grandes volumes de água doce (Nimer 1977).

Não foi observada associação entre as estações climáticas e a ocorrência da pitiose, diferente das demais regiões do país onde há maior ocorrência após estações chuvosas (Tabosa et al. 1999, Leal et al. 2001). Porém, a região sul diferencia-se do restante do país por apresentar clima com temperaturas bem demarcadas, invernos rigorosos, e chuvas bem distribuídas durante o ano todo (Nimer 1977).

Através da relação entre o histórico dos casos e o tempo estimado da evolução da doença em laboratório (Pereira et al.2008), ou na observação de casos espontâneos (Marcolongo-Pereira et al. 2012) é possível inferir que a infecção ocorreu em todas as estações do ano, tanto no inverno como no verão, sugerindo que Pythium insidiosum possa se reproduzir e infectar em temperaturas mais baixas do que as que a literatura cita.

Tem sido mencionado que pitiose não apresenta predisposição relacionada ao sexo (Mendoza et al. 1996). Porém, quando comparada às demais doenças, fêmeas tiveram 4,18 vezes mais chances de desenvolver a doença. Weiblen et al. (2016) avaliando a sorologia para $P$. insidiosum em equinos de todo estado do Rio Grande do Sul também encontrou risco relativo 1,59 vezes maior em fêmeas (IC 95\% = 1,11-2,27). Sugere-se que, nesses casos, a associação ocorra porque no Rio Grande do Sul, as fêmeas são a maior parte da tropa (Costa et al. 2014) e são usadas em sua maioria para a função reprodutiva, sendo mantidas a campo por mais tempo que os machos, propiciando um maior período de exposição ao agente causador da pitiose.

Em números absolutos a raça Crioula foi a mais afetada pela pitiose. Todavia, essa é a principal raça utilizada no Rio Grande do Sul (Pierezan et al. 2009). Contudo, por ser bem conhecida na região, justamente por sua alta casuística, muitas vezes não é realizada a confirmação laboratorial, o que resulta em subdiagnóstico, dificultando o conhecimento da dinâmica de distribuição dessa enfermidade.

Com relação às micotoxicoses, a maioria dos casos de LEME foi registrada nos meses frios, havendo seis vezes mais chances dessa intoxicação ocorrer nas estações do outono e inverno, confirmando a associação da mesma às baixas temperaturas (Riet-Correa et al. 1982, Meireles et al. 1994). Afirma-se que a LEME não tem predisposição por sexo (Riet-Correa et al. 2013), em contrapartida no presente estudo machos tiveram 3,4 vezes mais chances de se intoxicar. Entretanto, assim como na pitiose, é necessário considerar que é pratica comum na região o uso de equinos machos para serviços, os quais ficam mais tempo estabulados recebendo rações, aumentando as chances de exposição e intoxicação por micotoxinas presentes na ração contendo milho.

Observou-se, também, que os equinos que desenvolveram LEME eram em sua maioria cruzas de baixo valor econômico, utilizados no segmento de cavalos de trabalho, que segundo Lima \& Cintra (2015) se caracterizam por animais alimentados com volumoso de beira de estrada ou pasto, milho e farelo de trigo. Eventualmente, utilizam ração de baixo custo, fabricada (batida) na própria propriedade. Essa baixa qualidade do concentrado também pode aumentar as chances de produção e consumo de micotoxinas. Na evolução temporal das micotoxicoses, foi observada uma aparente tendência na diminuição do número de casos, que pode ser atribuída à disseminação de conhecimentos sobre a enfermidade e suas causas, levando à um melhor manejo dos animais.

As micoses ocorreram com frequência baixa e oscilatória, não havendo associação entre o número de casos e as variáveis analisadas. No presente estudo, foi encontrado o complexo T. mentagrophytes como o principal agente causal das dermatofitoses equinas na região. Situação diversa da relatada na literatura, que cita Trichophyton equinum e Microsporum canis como os fungos que mais causam dermatofitose em equinos (Pereira et al. 2006, Chermette et al. 2008, Lyskova et al. 2015,). Uma importante implicação desse fato está na saúde pública, uma vez que o complexo T. mentagrophytes é uma espécie antropofílica muito mais comum e implicada em infecções humanas (Cafarchia et al. 2013, Costa et al. 1999, Silveira-Gomes et al. 2013), enquanto T. equinum é zoofílico e raramente ocorre em humanos (Chabasse \& Pihet 2008).

A rinosporidiose é uma doença de ocorrência esporádica no Brasil. Em estudo realizado no país, observou-se que o estado do Rio Grande do Sul teve a maior casuística (Londero et al. 1977), possivelmente em função da proximidade deste estado com a Argentina, área considerada endêmica para rinosporidiose equina (Leeming et al. 2007, Burgess et al. 2012). No presente estudo a rinosporidiose teve maior chance de ocorrer em equinos machos e nas estações de clima quente $(\mathrm{p}<0,05)$. Dentro das infecções com registros de ocorrência raras, estão as micoses uterinas, sabidamente sua ocorrência depende muito das características individuais e de manejo das éguas (Rantala et al. 2015).

\section{CONCLUSÕES}

A pitiose equina foi a doença mais prevalente, sendo endêmica na região sudeste do Rio Grande do Sul.

São estabelecidas taxas de ocorrência e alguns fatores de risco relacionados à ocorrência de doenças fúngicas em equinos na região estudada, assim como se destaca que as incidências encontradas nesse estudo podem estar subestimadas, uma vez que as doenças aqui observadas não são de notificação obrigatória, e algumas já são de conhecimento de veterinários e proprietários, que muitas vezes não fazem a confirmação laboratorial do diagnóstico.

\section{REFERÊNCIAS}

Blanco J.L. \& Garcia M.E. 2008. Immune response to fungal infections. Vet Immunol. Immunopathol. 125(1/2):47-70. http://dx.doi.org/10.1016/j. vetimm.2008.04.020. PMid:18565595.

Burgess H.J., Lockerbie B.P., Czerwinski S. \& Scott M. 2012. Equine laryngeal rhinosporidiosis in western Canada. J. Vet. Diagn. Invest. 24(4):777-780. http://dx.doi.org/10.1177/1040638712445773. PMid:22604769.

Cafarchia C., Figueredo L.A. \& Otranto D. 2013. Fungal diseases of horses. Vet. Microbiol. 167(1/2):215-234. http://dx.doi.org/10.1016/j. vetmic.2013.01.015. PMid:23428378.

CAST 2003. Mycotoxins: Risks in Plants Animal and Human Systems. Task Force Report no. 139. Council for Agricultural Science and Technology, Ames, IA

Chabasse D. \& Pihet M. 2008. Les dermatophytes: les diffilcultés du diagnostic mycologique. Revue Francophone des Laboratoires 406(406):29-38. http:// dx.doi.org/10.1016/S1773-035X(08)74522-2.

Chermette R., Ferreiro L. \& Guillot J. 2008. Dermatophytoses in animals. Mycopathologia 166(5/6):385-405. http://dx.doi.org/10.1007/s11046008-9102-7. PMid:18478363. 
Costa T.R., Costa M.R., Silva M.V., Rodrigues A.B., Fernandes O.F.L., Soares A.J. \& Silva M.R. 1999. Etiology and epidemiology in dermatophytosis in Goiânia, State of Goiás, Brazil. Revta Soc. Bras. Med. Trop. 32(4):367-371. http://dx.doi.org/10.1590/S0037-86821999000400006. PMid:10495665.

Costa E., Diehl G.N., Santos D.V. \& Silva A.P.S. 2014. Panorama da Equinocultura no Rio Grande do Sul. Informativo Técnico 5, Secretaria de Agricultura do Estado do Rio Grande do Sul.

Fisher M.C., Henk D.A., Briggs C.J., Brownstein J.S., Madoff L.C., McCraw S.L. \& Gurr S.J. 2012. Emerging fungal threats to animal, plant and ecosystem health. Nature 484(7393):186-194. http://dx.doi.org/10.1038/nature10947. PMid:22498624.

Leal A.B.M., Leal A.T., Santurio J.M., Kommers G.D. \& Catto J.B. 2001. Pitiose equina no pantanal brasileiro: aspectos clínico-patológico de casos típicose atípicos. Pesq. Vet. Bras. 21(4):151-156. http://dx.doi.org/10.1590/ S0100-736X2001000400005.

Leeming G., Smith K.C., Bestbier M.E., Barrelet A. \& Kipar A. 2007. Equine rhinosporidiosis in United Kingdom. Emerg. Infect. Dis. 13(9):1377-1379. http://dx.doi.org/10.3201/eid1309.070532. PMid:18252114.

Lima R.A.D.S. \& Cintra A.G. 2015. Revisão Estudo do Complexo do Agronegócio Cavalo. MAPA, Brasília.

Londero A.T., Santos M.N. \& Freitas C.J.B. 1977. Animal rhinosporidiosis in Brazil: report of three additional cases. Mycopathologia 60(3):171-173. http://dx.doi.org/10.1007/BF00448411. PMid:559253.

Lyskova P., Hubka V., Petricakova A., Dobias R., Cmokova A. \& Kolarik M. 2015. Equine dermatophytosis due to Trichophyton bullosum, a poorly known zoophilic dermatophyte masquerading as T. verrucosum. Mycopathologia 180(5/6):407-419. http://dx.doi.org/10.1007/s11046-015-9931-0. PMid:26290003.

Marcolongo-Pereira C., Sallis E.S.V., Raffi M.B., Pereira D.I.B., Hinnah F.L., Coelho A.C.B. \& Schild A.L. 2012. Epidemiologia da pitiose equina na Região Sul do Rio Grande do Sul. Pesq. Vet. Bras. 32(9):865-868. http://dx.doi. org/10.1590/S0100-736X2012000900009.

Marcolongo-Pereira C., Estima-Silva P., Soares M.P., Sallis E.S.V., Grecco F.B., Raffi M.B., Fernandes C.G. \& Schild A.L. 2014. Doenças de equinos na região Sul do Rio Grande do Sul. Pesq. Vet. Bras. 34(3):205-210. http://dx.doi org/10.1590/S0100-736X2014000300002.

Meireles M.C.A., Corrêa B., Fischman O., Gambale W., Paula C.R., ChaconReche N.O. \& Pozzi C.R. 1994. Mycoflora of the toxic feeds associated with equine leucoencephalomalacia (ELEM) outbreaks in Brazil. Mycopathology 117:183-188. http://dx.doi.org/10.1007/BF01102919.

Mendoza L., Ajello L. \& McGinnis M.R. 1996. Infections caused by the oomycetous pathogen Pythium insidiosum. J. Mycologie Médicale 6:151-164.
Miller R.I. \& Campbell R.S.F. 1982. Clinical observations on equine phycomycosis. Aust. Vet. J. 58(6):221-226. http://dx.doi.org/10.1111/j.1751-0813.1982. tb00681.x. PMid:6890342.

Nimer E. 1977. Clima, p.35-79. In: IBGE (Ed.), Geografia do Brasil, Região Sul. Sergraf-IBGE, Rio de Janeiro.

Pereira D.I.B., Oliveira L.S.S., Bueno A., Cavalheiro A.S., Schwendler S.E., Azevedo M.I., Eckhardt Júnior J.C., Aguiar L.C., Santurio D.F., Santurio J.M. \& Alves S.H. 2006. Surto de Trichophyton equinum var. equinum em equinos no sul do Brasil. Ciência Rural 36(6):1849-1853. http://dx.doi. org/10.1590/S0103-84782006000600028.

Pereira D.I.B., Santurio J.M., Alves S.H., Azevedo M.I., Silveira F., Costa F.F., Sallis E.S.V., Pötter L. \& Ferreiro L. 2008. Comparison between the immunotherapic Pitium Vac and the antifungal drug caspofungin as agents to treat experimental pythiosis in rabbits. J. Mycologie Médicale 18:129133. http://dx.doi.org/10.1016/j.mycmed.2008.05.001.

Pierezan F., Rissi D.R., Rech R.R., Fighera R.A., Brum J.S. \& Barros C.S.L. 2009. Achados de necropsia relacionados com a morte de 335 equinos: 1968-2007. Pesq. Vet. Bras. 29(3):275-280. http://dx.doi.org/10.1590/ S0100-736X2009000300015.

Rantala M., Attia S., Koukila-Kähkölä P., Hoog S., Anttila M. \& Katila T. 2015. Cladophialophora bantiana as an emerging pathogen in animals: case report of equine endometritis and review of the literature. J. Clin. Microbiol. 53(9):3047-3053. http://dx.doi.org/10.1128/JCM.00728-15. PMid:26085616.

Riet-Correa F., Meirelles M.A., Soares J.M., Machado J.J. \& Zambrano A.F. 1982. Leucoencefalomalácia em equinos associado à ingestão de milho mofado. Pesq. Vet. Bras. 2:27-30.

Riet-Correa F., Rivero R., Odriozola E., Adrien M.L., Medeiros R.M. \& Schild A.L. 2013. Mycotoxicoses of ruminants and horses. J. Vet. Diagn. Invest. 25(6):692708. http://dx.doi.org/10.1177/1040638713504572. PMid:24091682.

Scott D.W. \& Miller W.H. 2011. Equine Dermatology, 2nd ed. Elsevier Saunders, Maryland Heights.

Silveira-Gomes F., Oliveira E.F., Nepomuceno L.B., Pimentel R.F., Marques-daSilva S.H. \& Mesquita-da-Costa M. 2013. Dermatophytosis diagnosed at the Evandro Chagas Institute, Pará, Brazil. Braz. J. Microbiol. 44(2):443-446. http://dx.doi.org/10.1590/S1517-83822013005000049. PMid:24294235.

Tabosa I.M., Medeiros V.T., Dantas A.F.M., Azevedo E.O. \& Maia J.C. 1999. Pitiose cutânea em equídeos no semi-árido da Paraíba. Arq. Bras. Med. Vet. Zootec. 51:27-30.

Weiblen C., Machado G., Jesus F.P.K., Santurio J.M., Zanette R.A., Pereira D.I.B., Diehl G.N., Santos L.C., Corbellini L.G. \& Botton S.A. 2016. Seroprevalence of Pythium insidiosum infection in equine in Rio Grande do Sul, Brazil. Ciência Rural 46(1):126-131. http://dx.doi.org/10.1590/0103-8478cr20150056. 